\title{
Estrutura da Vegetação Caducifólia Espinhosa (Caatinga) de uma área do sertão central de Pernambuco
}

\author{
Maria Jesus Nogueira Rodal ${ }^{1,3}$, Keila Cristina Carvalho Costa $^{2}$ e Ana Carolina Borges Lins e Silva ${ }^{1}$
}

Recebido: 20.04.2006; aceito: 30.05.2008

ABSTRACT - (Vegetation structure of the thorny deciduous woodland (Caatinga) in an area of central sertão of Pernambuco). Vegetation structure of a "Caatinga" sensu stricto (thorny deciduous woodland) was studied in a lowland area at the municipalities of Floresta and Betânia, State of Pernambuco, Brazil. Height and perimeter of all woody plants to be found in 1 hectare were censured when stem perimeter at soil level was $\geq 3 \mathrm{~cm}$ and the height $\geq 1 \mathrm{~m}$. A total of 3,140 individuals belonging to 28 plant species were registered with a basal area of $18.5 \mathrm{~m}^{2}$. In general, results from surveys at lowlands on plain areas presented smaller individual stem diameters than those located near streams or mountain range sites. Physiognomic differences in the caatinga sensu stricto on lowlands are related to differences on the density of plants with greater diameters. The diameter distribution of Caesalpinia gardneriana Benth. (Caesalpiniaceae) and Croton rhamnifolioides Pax \& K. Hoffm. (Euphorbiaceae), the two most important tree species, indicate that these plant populations may be regenerating from former disturbance.

Key words: "Sertaneja" depression, structure, woody plants

RESUMO - (Estrutura da vegetação caducifólia espinhosa (Caatinga) de uma área do sertão central de Pernambuco). Foi realizada a descrição da estrutura da vegetação de caatinga sensu stricto do pediplano da depressão sertaneja situada entre os municípios de Floresta e Betânia, Pernambuco. Foram amostrados todos os indivíduos vivos com diâmetro do caule ao nível do solo $\geq 3 \mathrm{~cm}$ e altura total $\geq 1 \mathrm{~m}$ presentes em um hectare. Foram registradas 28 espécies, um total de 3.140 plantas com área basal total de $18,5 \mathrm{~m}^{2}$. Em geral, os levantamentos da depressão sertaneja, situados em áreas do pediplano, apresentam menores diâmetros que aqueles situados em áreas próximas a riachos ou serras. É possível concluir que as diferenças fisionômicas na caatinga sensu stricto da depressão ocorrem na função da densidade das plantas com maiores diâmetros. As distribuições diamétricas de Caesalpinia gardneriana Benth. (Caesalpiniaceae) e Croton rhamnifolioides Pax \& K. Hoffm. (Euphorbiaceae), espécies de maior valor de importância, indicam populações em regeneração.

Palavras-chave: depressão sertaneja, estrutura, plantas lenhosas

\section{Introdução}

Das províncias biogeográficas presentes no território brasileiro (sensu Cabrera \& Willink 1973), a da Caatinga, com aproximadamente os mesmos limites da área de clima semi-árido do nordeste brasileiro, é uma das maiores e mais desconhecidas. Sua variada cobertura vegetal está, em grande parte, determinada pelo clima, relevo e embasamento geológico que, em suas múltiplas interrelações, resultam em ambientes ecológicos bastante variados. A heterogeneidade da flora e da fisionomia da cobertura vegetal dessa província decorre de dois gradientes de umidade, um no sentido Norte-Sul, que se manifesta em uma diminuição das precipitações e outro Oeste - Leste, que se expressa com um aumento do efeito da continentalidade. Além disso, as variações topográficas que ocorrem no interior dessa província contribuem para a ocorrência de gradientes menores. Um outro fator importante na diversificação vegetacional, especialmente na flora local, é a história dos eventos geológicos presentes em cada uma das unidades vegetacionais.

De acordo com Veloso et al. (1991), a savanaestépica é a tipologia vegetal característica e de maior extensão daquela província, sendo localmente chamada de caatinga. A savana-estépica, doravante denominada caatinga sensu stricto, ocorre especialmente nas terras

1. Universidade Federal Rural de Pernambuco, Departamento de Biologia, Avenida Dom Manoel de Medeiros s/n, Dois Irmãos, 52171900 Recife, PE, Brasil

2. Instituto de Desenvolvimento Científico e Tecnológico do Xingó, 49820-000 Canindé de São Francisco, SE, Brasil

3. Autor para correspondência: mrodal@terra.com.br 
baixas entre serras e planaltos, a chamada depressão sertaneja, a qual ocupa $368.216 \mathrm{~km}^{2}$ (para revisão ver Souza et al. 1994). A depressão representa um extenso conjunto de pediplanos ora rodeado por extensos planaltos como o da Ibiapaba, entre o Piauí e o Ceará, ora entremeado por relevos residuais com variadas dimensões como chapadas e bacias sedimentares, maciços e serras (Rodal \& Sampaio 2002).

Embora Alcoforado Filho et al. (2003) afirmem que a vegetação de caatinga sensu stricto é o tipo vegetacional mais estudado na área da depressão sertaneja, ainda não há uma visão compreensiva das variações fisionômicas dessa vegetação em razão dos estudos apresentarem uma grande diversidade metodológica, fruto de seus diferentes objetivos (para uma revisão dos levantamentos quantitativos ver Sampaio 1996). Além disso, a maioria dos trabalhos não define de forma clara a questão do grau de perturbação.

As descrições de caatinga apresentadas por autores como Andrade-Lima (1981) e Sampaio (1995) apontam na direção de que, em uma mesma região, as diferenças fisionômicas encontradas na vegetação de caatinga sensu stricto estão relacionadas com as variações ambientais como a proximidade de serras e corpos de água.

Com objetivo de contribuir para o conhecimento da vegetação lenhosa de caatinga sensu stricto situada da depressão, foi realizada a caracterização da vegetação lenhosa e a análise da distribuição diamétrica de todas as plantas amostradas e de algumas populações. Adicionalmente, foi realizada a comparação da distribuição de diâmetros e alturas da área de estudo com a encontrada com dois habitas típicos caatinga da depressão, as áreas do pediplano e as áreas próximas a riachos e serras.

\section{Material e métodos}

Área de estudo - A Reserva Natural do Patrimônio Particular (RPPN) Maurício Dantas (8¹8'43"S e $\left.38^{\circ} 11^{\prime} 45^{\prime} \mathrm{W}\right)$, localizada entre os municípios de Floresta e Betânia, Pernambuco, a cerca de 420 km da capital Recife, ocupa 1.485 hectares em uma área do pediplano situada em plena depressão sertaneja. O levantamento fitossociológico foi realizado em uma área com $545 \mathrm{~m}$ de altitude. As chuvas são concentradas entre fevereiro e abril, com precipitação e temperatura médias anuais de $511 \mathrm{~mm}$ e $25^{\circ} \mathrm{C}$, respectivamente (Instituto Nacional de Meteorologia (2006) (figura 1).
Os terrenos da RPPN têm litologia predominantemente composta por rochas précambrianas, em alguns trechos recobertos por chapadas residuais (Brasil 1983). Os solos predominantes da região são uma associação de Planossolo, Solonetz solodizado, solos Litólicos eutróficos, Regossolos eutróficos e distróficos e Bruno não cálcico (Embrapa 2003). Os valores médios das determinações físicas e químicas (Embrapa 1997) de amostras do solo tiradas na área de amostragem, na profundidade de 0 a $20 \mathrm{~cm}$, foram: areia, silte e argila, 37,6, 14,6 e $47,8 \%$ respectivamente; umidade a $1 / 3$ atm e $15 \mathrm{~atm}$, 23,34 e 17,75\%; pH em água, 7,37; $\mathrm{Ca}, \mathrm{Mg}, \mathrm{H}+\mathrm{e} \mathrm{Al}$ trocáveis, $21,01,8,75,1,50 \mathrm{cmol}_{\mathrm{c}} \mathrm{kg}^{-1}$ e saturação de bases de $96,68 \%$.

Coleta e tratamento de dados - Para o levantamento quantitativo da flora lenhosa foi instalada uma parcela de $100 \mathrm{~m} \times 100 \mathrm{~m}$, subdividida em sub-parcelas de $10 \mathrm{~m} \times 10 \mathrm{~m}$, em uma área do pediplano da RPPN com poucos sinais de ação antrópica, onde, segundo informações dos moradores locais, não houve interferência há pelo menos 20 anos. Foram amostrados e coletados os indivíduos vivos com diâmetro do caule ao nível do solo $\geq$ a $3 \mathrm{~cm}$ e altura total e $\geq 1 \mathrm{~m}$ (Rodal et al. 1992). Foram calculados parâmetros gerais da comunidade (densidade total e área basal totais e no caso da altura e diâmetro, calculados os valores médios, acompanhados do desvio padrão), parâmetros relativos dos táxons (densidade, frequiência, área basal, valor de importância) (Mueller-Dombois \& Ellenberg 1974). Os cálculos foram realizados usando o FITOPAC (Shepherd 1994).

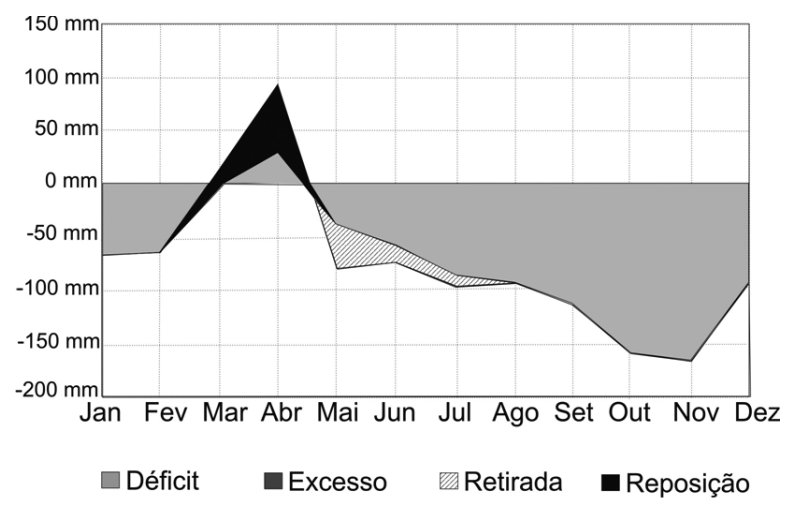

Figura 1. Balanço hídrico climatológico usando capacidade de armazenamento de água no solo de $100 \mathrm{~mm}$. O gráfico foi adquirido do site do Instituto Nacional de Metereologia do Ministério da Agricultura, registrados no posto metereológico do Município de Floresta no período de 30 anos (1961-1990). 
As identificações ocorreram com base em bibliografia especializada, por especialistas e por comparação com material depositado no herbário Professor VasconcelosSobrinho (PEUFR) da Universidade Federal Rural de Pernambuco. O sistema de classificação de Cronquist (1981) foi adotado para este trabalho.

Com objetivo de caracterizar a ocupação do espaço vertical, foram analisados o número de indivíduos e área basal por classe de altura com intervalo de $1 \mathrm{~m}$. Em seguida, foi calculado o número de indivíduos nas classes de diâmetro de caule de 3-12, > 12-27 e > $27 \mathrm{~cm}$ e na classe de altura superior a $8 \mathrm{~m}$, seguindo a metodologia descrita em Alcoforado Filho et al. (2003). Em seguida, foi realizada uma revisão dos levantamentos localizados na depressão sertaneja (tabela 1), sendo selecionados aqueles que disponibilizavam informações a respeito das classes de diâmetro e altura citadas e a localização de cada levantamento, isto é, se no pediplano (Rodal 1992, Araújo et al. 1995, Medeiros 1995, Ferraz et al. 2003) ou em áreas próximas a riachos/ serras (Rodal 1992, Araújo et al. 1995, Ferraz et al. 2003). Com base nesses dados, foram comparadas as médias de diâmetro e altura entre as duas situações (pediplano e serras/ riachos), empregando o teste de Mann-Whitney através do programa BIOSTAT 2.0 (Ayres et al. 2000).

Foi calculada a distribuição diamétrica para a amostra como um todo e para aquelas populações com mais de 100 indivíduos (exceto cactáceas), por considerarmos esse tamanho mais representativo para avaliar a estrutura diamétrica dessas populações. $\mathrm{O}$ intervalo de classe empregado foi obtido através da fórmula: número de classes $(\mathrm{nc})=1+3,3^{*} \log \left(\mathrm{n}^{\mathrm{o}}\right.$ ind.) ; com o intervalo de classes calculado pela amplitude (maior - menor valor de diâmetro) dividido pelo número de classes (Nascimento et al. 2004).

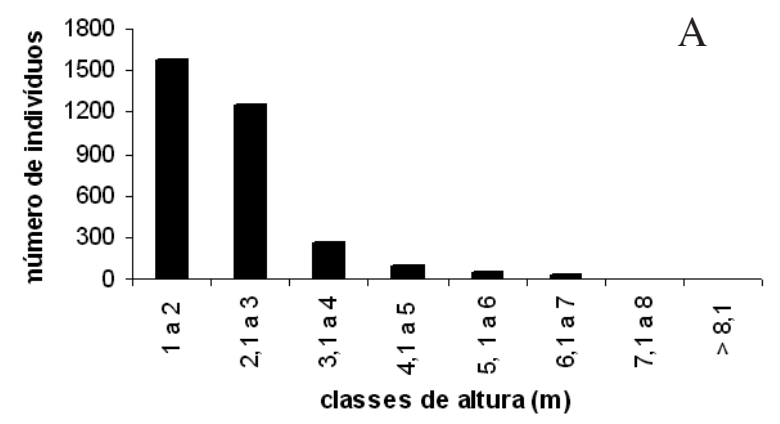

\section{Resultados}

Foram amostrados 3.140 indivíduos vivos, correspondendo a uma área basal total (ABT) de 18,5 $\mathrm{m}^{2} \mathrm{ha}^{-1}$. As alturas e diâmetros médios foram: $2,37 \mathrm{~m}$ $( \pm 0.925), 7,3 \mathrm{~cm}( \pm 4.655)$. A altura máxima foi 12 m e o maior diâmetro $45,68 \mathrm{~cm}$.

Os resultados de altura, diâmetro e área basal mostraram que: (1) cerca de $50 \%$ dos indivíduos apresentaram altura $\geq 2 \mathrm{~m}$; (2) o maior valor de área basal total (ABT) ocorreu na classe de altura de 2,1 a $3 \mathrm{~m}$, diminuindo gradativamente, até $6 \mathrm{~m}$ para depois cair bruscamente; (3) os indivíduos com até $3 \mathrm{~m}$ responderam por $63 \%$ da ABT; e (4) mais de $50 \%$ dos indivíduos tiveram diâmetro do caule inferior a $6 \mathrm{~cm}$ (figuras 2, 3A). A distribuição do total de indivíduos por classe de diâmetro indica que a amostra apresenta padrão de $\mathrm{J}$ invertido, indicando uma comunidade em regeneração (figura 3A).

Fisionomicamente, a área basal registrada na área de estudo esteve entre as mais baixas já listadas para a depressão sertaneja, exceto duas das quatro áreas estudadas por Rodal (1992) e por Camacho (2001) (tabela 1). Os valores mais elevados de área basal ocorreram em áreas próximas a riachos (Rodal 1992) ou a serras (Ferraz et al. 2003) (tabela 1).

Comparando os resultados com outros levantamentos em termos número de indivíduos em classes de diâmetro e número de indivíduos com mais de $8 \mathrm{~m}$ de altura (tabela 2), nota-se que aqueles situados em áreas do pediplano deferiram daqueles efetuados em áreas próximas a riachos/ serras pela maior altura e pela densidade das plantas com mais de $27 \mathrm{~cm}$ de circunferência.

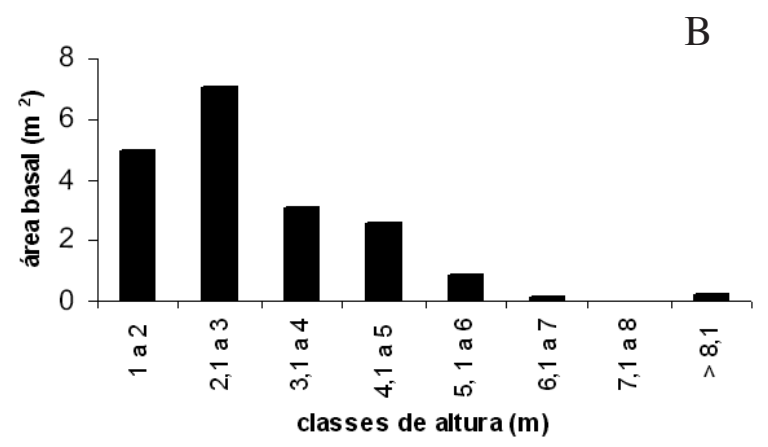

Figura 2. Número de indivíduos (A) e área basal (B) por classes de altura na Reserva Particular do Patrimônio Natural Maurício Dantas, Pernambuco. 

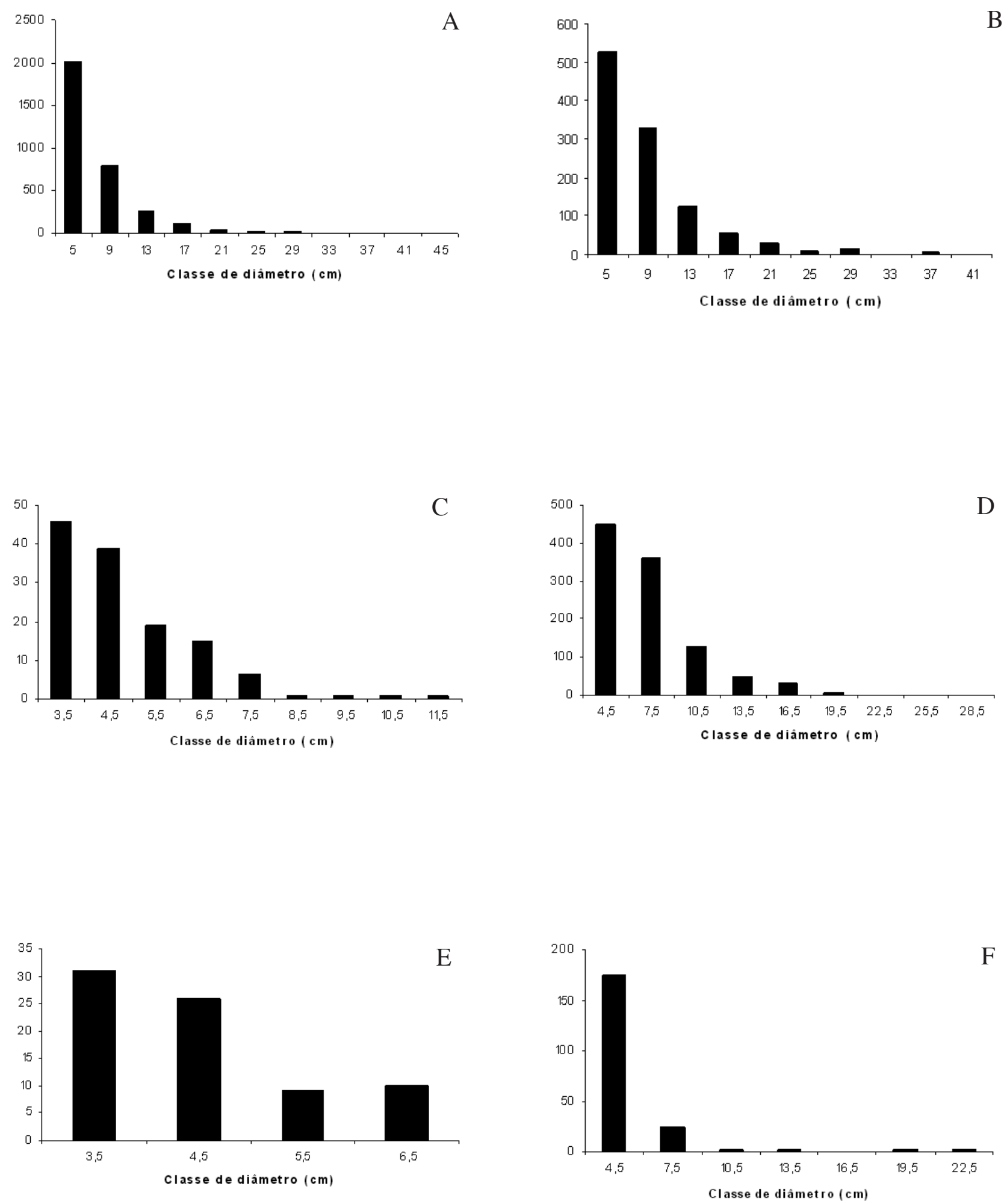

Figura 3. Distribuição diamétrica de algumas espécies amostradas na Reserva Particular do Patrimônio Natural Maurício Dantas, Pernambuco. $\mathrm{A}=$ total de indivíduos da amostra, $\mathrm{B}=$ Caesalpinia gardneriana Benth., $\mathrm{C}=$ Croton blanchetianus Müll. Arg., $\mathrm{D}=$ C. rhamnifolioides Pax \& K. Hoffm., E = Jatropha mollissima (Pohl) Baill. e F = Aspidosperma pyrifolium Mart. Os intervalos estão representados pelo valor central de cada classe. 
O número de espécies amostradas (28) foi semelhante ao de outros levantamentos quantitativos de plantas lenhosas em caatinga, exceto para as localidades estudadas por Camacho (2001) (tabela 1). Duas das espécies com maior importância estrutural - Caesalpinia gardneriana e Croton rhamnifolioides -, apresentaram valores semelhantes em termos de densidade e frequiência diferindo em termos de dominância, com a primeira tendo praticamente o dobro da segunda.

A dominância foi um parâmetro importante na composição do VI de espécies arbóreas como Commiphora leptophloeos, Myracrodruon urundeuva e Schinopsis brasiliensis. Por outro lado, arvoretas como Aspidosperma pyrifolium e Cnidoscolus quercifolius, tiveram seu VI associado aos maiores valores de densidade e freqüência na primeira espécie e freqüência e dominância na segunda.

A altura média de $2,37 \mathrm{~m}$ foi fortemente influenciada pelo grande número de arbustos de Croton rhamnifolioides e $C$. blanchetianus cujas alturas médias e máximas foram 1,5 m, 2,5 m e 1,9 m e $2 \mathrm{~m}$, respectivamente. Essas espécies respondem por $73 \%$ dos indivíduos amostrados na classe entre 1 e $2 \mathrm{~m}$. A maior concentração de ABT entre 2,1 e $3 \mathrm{~m}$ ocorreu basicamente em função da arvoreta de pequeno porte Caesalpinia gardneriana, que respondeu por $65 \%$ da área basal daquela classe. Das espécies amostradas, apenas Caesalpinia gardneriana, Schinopsis brasiliensis e Cnidoscolus quercifolius apresentaram indivíduos maiores que $8 \mathrm{~m}$.
A figura 3 mostra a distribuição diamétrica do hectare e das espécies com mais 100 indivíduos, exceto as da família Cactaceae. Aparentemente, o padrão de $\mathrm{J}$ invertido se deveu basicamente ao comportamento das duas espécies de maior VI, Caesalpinia gardneriana e Croton rhamnifolioides, e a C. blanchetianus e Jatropha mollissima (tabela 3), todas com elevado numero de indivíduos nas classes de menor diâmetro.

Caesalpinia gardneriana (figura 3B), Croton rhamnifolioides (figura 3C), Croton blanchetianus (figura 3D) e Jatropha mollissima (figura 3E) apresentaram distribuição diamétrica contínua, com diminuição progressiva do número de indivíduos da menor para a maior classe de diâmetro. Em Aspidosperma pyrifolium (figura 3F) quase todos os indivíduos ocorrem na primeira classe, com alguns poucos em maiores classes.

O baixo número de indivíduos de espécies arbóreas como Amburana cearensis, Anadenanthera colubrina, Commiphora leptophloeos, Myracrodruon urundeuva e Schinopsis brasiliensis (tabela 3) não permitiu maiores inferências a respeito da distribuição diamétrica.

\section{Discussão}

Os resultados da tabela 1 mostraram que as duas situações diferiram apenas em termos da densidade

Tabela 1. Metodologia e parâmetros fisionômicos da área de estudo situada na Reserva Particular do Patrimônio Natural Maurício Dantas, Pernambuco e de outros levantamentos quantitativos de caatinga sensu stricto na depressão sertaneja no nordeste do Brasil. CI = critério de inclusão, PNS = perímetro do caule ao nível do solo; DNS = diâmetro do caule ao nível do solo; $\mathrm{AA}=$ área amostrada $\left(\mathrm{m}^{2}\right)$; $\mathrm{pq}=$ ponto quadrante; $\mathrm{NE}=$ número de espécies; $\mathrm{DT}=$ densidade total (ind. ha $\left.{ }^{-1}\right) ; \mathrm{ABT}=$ área basal total $\left(\mathrm{m}^{2} \mathrm{ha}^{-1}\right) ; \mathrm{Alt} .=$ altitude $(\mathrm{m})$; Prec. $=$ precipitação $\left(\mathrm{mm}\right.$ ano $\left.{ }^{-1}\right)$.

\begin{tabular}{|c|c|c|c|c|c|c|}
\hline Autor/ município /UF & $\mathrm{CI}$ & AA & $\mathrm{NE}$ & DT & $\mathrm{ABT}$ & Prec. \\
\hline Rodal (1992) - Custódia1, PE & $\mathrm{DNS} \geq 3 \mathrm{~cm}$ & 2500 & 22 & 1076 & 34,3 & 651 \\
\hline Rodal (1992) - Custódia2, PE & $\mathrm{DNS} \geq 3 \mathrm{~cm}$ & 2500 & 28 & 1872 & 20,3 & 651 \\
\hline Rodal (1992) - Floresta1, PE & $\mathrm{DNS} \geq 3 \mathrm{~cm}$ & 2500 & 23 & 1876 & 16,5 & 632 \\
\hline Rodal (1992) - Floresta2, PE & $\mathrm{DNS} \geq 3 \mathrm{~cm}$ & 2500 & 24 & 2172 & 15,6 & 632 \\
\hline Araújo et al. (1995) - Floresta3, PE & $\mathrm{PNS} \geq 5 \mathrm{~cm}$ & $100 \mathrm{pq}$ & 27 & 3023 & 19,8 & 586 \\
\hline Araújo et al. (1995) - Floresta4, PE & $\mathrm{PNS} \geq 5 \mathrm{~cm}$ & 100 pq & 22 & 5385 & 31,1 & 586 \\
\hline Araújo et al. (1995) - Custódia3, PE & $\mathrm{PNS} \geq 5 \mathrm{~cm}$ & $100 \mathrm{pq}$ & 25 & 3975 & 32,2 & 574 \\
\hline Camacho (2001) - Mossoró1, RN & $\mathrm{DNS} \geq 3 \mathrm{~cm}$ & 5000 & 12 & 6320 & 19,9 & 497 \\
\hline Camacho (2001) - Mossoró2, RN & $\mathrm{DNS} \geq 3 \mathrm{~cm}$ & 5000 & 13 & 7015 & 24,5 & 497 \\
\hline Camacho (2001) - Mossoró3, RN & DNS $\geq 3 \mathrm{~cm}$ & 5000 & 11 & 4120 & 9,5 & 497 \\
\hline Camacho (2001) - Mossoró4, RN & $\mathrm{DNS} \geq 3 \mathrm{~cm}$ & 5000 & 9 & 2812 & 12,4 & 497 \\
\hline Ferraz et al. (2003) - 700 m, Serra Talhada1, PE & $\mathrm{DNS} \geq 3 \mathrm{~cm}$ & 1000 & 22 & 5590 & 52,4 & 874 \\
\hline Ferraz et al. (2003) - 500 m, Serra Talhada2, PE & $\mathrm{DNS} \geq 3 \mathrm{~cm}$ & 2000 & 35 & 3555 & 30,6 & 679 \\
\hline
\end{tabular}


das plantas com mais de $27 \mathrm{~cm}$ de diâmetro (tabela 2), indicando que a fisionomia de caatinga sensu stricto da depressão sertaneja caracteriza-se por uma grande densidade de plantas de pequeno porte e altura e que as diferenças fisionômicas ocorrem em função da presença de indivíduos com maiores circunferências.

Das espécies presentes no hectare, sete não foram listadas nos demais levantamentos da depressão sertaneja (tabela 1): Arrojadoa rhodantha, Caesalpinia gardneriana, Erythroxylum pungens, Croton rhamnifolioides, Guapira noxia, Senna macranthera. Como a análise das etiquetas das exsicatas dessas espécies nos herbários IPA, PEUFR e UFP mostra sua ocorrência em diversas áreas da depressão sertaneja, é possível que a explicação para essas ausências se deva à identificação incompleta. Vale ressaltar que alguns desses grupos taxonômicos só recentemente foram revisados como: Caesalpinia grupo Poincianella-Erythrostemon (Lewis 1998) e Erythroxylum sect. Rhabdophyllum (Loiola 2001) ou ainda melhor estudados na região como Croton (Lucena 2001).

Como exemplo desse desconhecimento, hoje sabe-se que Caesalpinia pyramidalis, a espécie mais freqüente nos levantamentos de caatinga (Sampaio 1996), na verdade representa com complexo de três espécies e diversas subespécies com padrões de distribuição geográfica diferenciados (Lewis 1998). Assim, o fato de as espécies de maior importância na área não terem sido relacionadas entre as mais importantes em outros levantamentos em áreas de caatinga sensu stricto provavelmente se deve a problemas de identificação de material, como Croton rhamnifolioides (Euphorbiaceae) ou Caesalpinia gardneriana (Caesalpiniaceae).

Esses dados indicam que mesmo o componente lenhoso da flora da caatinga sensu stricto, o mais estudado, é ainda pouco conhecido. Tais resultados sugerem que apesar da baixa riqueza de espécies lenhosas por unidade de área na caatinga sensu stricto (Rizzini 1979), muito ainda deve ser feito para ampliar o conhecimento taxonômico de diferentes grupos botânicos dessa vegetação.

O padrão de distribuição diamétrica encontrado em Caesalpinia gardneriana, Croton rhamnifolioides, C. blanchetianus e Jatropha mollissima, revela uma diminuição progressiva do número de indivíduos em direção das maiores classes de diâmetro. Todavia, essa distribuição contínua não significa necessariamente

Tabela 2. Resultados do teste de Mann-Whitney de comparação das médias do número de plantas por hectare em classes de diâmetro e altura entre levantamentos de dois tipos de habitats da depressão (próximos a riachos ou serra e situados no pediplano) no nordeste do Brasil, empregando o teste de com informações do município, unidade da federação e referência.

\begin{tabular}{|c|c|c|c|c|c|}
\hline \multirow[t]{2}{*}{ Município/ UF } & \multicolumn{2}{|c|}{ Diâmetro (cm) } & \multicolumn{2}{|c|}{ Altura (m) } & \multirow[t]{2}{*}{ Referência } \\
\hline & $3-12$ & $>12-27$ & $>27$ & $>8$ & \\
\hline \multicolumn{6}{|c|}{ Levantamentos na depressão próximos a riachos ou serras } \\
\hline Capistrano, CE & 2802 & 305 & 69 & 552 & Medeiros (1995) \\
\hline Custódia 1, PE & 1596 & 272 & 60 & 212 & Rodal (1992) \\
\hline Custódia 2, PE & 748 & 176 & 156 & 24 & Rodal (1992) \\
\hline Custódia 3, PE & 2128 & 564 & 89 & 238 & Araújo et al. (1995) \\
\hline Serra Talhada 1, PE & 2800 & 610 & 180 & 50 & Ferraz et al. (2003) \\
\hline Serra Talhada 2, PE & 3025 & 410 & 120 & 85 & Ferraz et al. (2003) \\
\hline Média & 2183 & 389 & 112 & 193 & \\
\hline \multicolumn{6}{|c|}{ Levantamentos na depressão situados no pediplano } \\
\hline Floresta 1, PE & 1552 & 268 & 56 & 56 & Rodal (1992) \\
\hline Floresta 2, PE & 1872 & 268 & 32 & 28 & Rodal (1992) \\
\hline Floresta 3, PE & 3415 & 364 & 67 & 0 & Araújo et al. (1995) \\
\hline Floresta 4, PE & 1763 & 319 & 61 & 0 & Araújo et al. (1995) \\
\hline Área de estudo, PE & 2723 & 381 & 33 & 3 & Este trabalho \\
\hline \multirow[t]{2}{*}{ Média } & $2265 \mathrm{NS}$ & $394 \mathrm{NS}$ & $50 *$ & $17 *$ & \\
\hline & $\mathrm{P}=0,8551$ & $\mathrm{P}=0,4652$ & $\mathrm{P}=0,0176$ & $\mathrm{P}=0,0285$ & \\
\hline
\end{tabular}

* e NS significam diferenças significativas a 5\% e não significativas pelo teste de Mann-Whitney. 
Tabela 3. Parâmetros fitossociológicos das espécies amostradas em um hectare da Reserva Particular do Patrimônio Natural Maurício Dantas, Pernambuco, em ordem decrescente do índice do valor importância por táxon (VIt). NC = número do coletor, NI = número de indivíduos, $\mathrm{NP}=$ frequiência absoluta do táxon $(\%), \mathrm{DRt}=$ densidade relativa do táxon $(\%)$; DoRt = dominância relativa do táxon (\%); $\mathrm{FRt}=$ freqüência relativa do táxon $(\%)$. O material botânico está tombado no herbário PEUFR. AC = Ana Carolina Borges Lins e Silva, $\mathrm{KC}=$ Keila Cristina Carvalho Costa, LP = Luciana Maranhão Pessoa, MR = Maria Jesus Nogueira Rodal

\begin{tabular}{|c|c|c|c|c|c|c|c|}
\hline Espécies (famílias) & $\mathrm{NC}$ & NI & NP & DRt & DoRt & FRt & VIt \\
\hline Caesalpinia gardneriana Benth. (Caesalpiniaceae) & MR 875 & 1099 & 99 & 34,8 & 49,4 & 14,7 & 99,0 \\
\hline Croton rhamnifolioides Pax \& K. Hoffm. (Euphorbiaceae) & $\mathrm{AC} 7$ & 1029 & 92 & 32,6 & 26,4 & 13,7 & 72,6 \\
\hline Opuntia palmadora Britton \& Rose (Cactaceae) & KC 276 & 197 & 73 & 6,3 & 2,0 & 10,8 & 19,0 \\
\hline Aspidosperma pyrifolium Mart. (Apocynaceae) & MR 829 & 203 & 69 & 6,4 & 2,3 & 10,2 & 18,9 \\
\hline Cnidosculus quercifolius Pohl (Euphorbiaceae) & KC 290 & 98 & 63 & 3,1 & 4,1 & 9,4 & 16,5 \\
\hline Jatropha mollissima (Pohl) Baill. (Euphorbiaceae) & KC 243 & 138 & 73 & 4,4 & 1,1 & 10,8 & 16,3 \\
\hline $\begin{array}{l}\text { Pilosocereus gounellei (F.A.C. Weber) Byles \& G.D. Rowley } \\
\text { (Cactaceae) }\end{array}$ & LP 310 & 83 & 43 & 2,6 & 3,6 & 6,4 & 12,6 \\
\hline Croton blanchetianus Müll. Arg. (Euphorbiaceae) & LP 9 & 131 & 25 & 4,2 & 1,4 & 3,7 & 9,2 \\
\hline Cnidosculus bahianus (Ule) Pax \& K. Hoffm. (Euphorbiaceae) & KC 417 & 53 & 35 & 1,7 & 1,2 & 5,2 & 8,1 \\
\hline Mimosa ophtalmocentra Mart. ex Benth. (Mimosaceae) & KC 258 & 40 & 29 & 1,3 & 0,5 & 4,3 & 6,0 \\
\hline Schinopsis brasiliensis Engl. (Anacardiaceae) & KC 404 & 9 & 7 & 0,3 & 3,8 & 1,0 & 5,1 \\
\hline Cereus jamacaru DC. (Cactaceae) & KC 393 & 11 & 11 & 0,4 & 0,6 & 1,6 & 2,6 \\
\hline Commiphora leptophloeos (Burseraceae) & KC 275 & 3 & 3 & 0,1 & 1,3 & 0,5 & 1,8 \\
\hline Piptadenia stipulacea (Benth.) Ducke (Mimosaceae) & KC 235 & 10 & 9 & 0,3 & 0,1 & 1,3 & 1,7 \\
\hline Manihot sp. (Euphorbiaceae) & KC 238 & 8 & 8 & 0,3 & 0,2 & 1,2 & 1,7 \\
\hline Arrojadoa rhodantha (Gürke) Britton \& Rose (Cactaceae) & MR 822 & 10 & 7 & 0,3 & 0,1 & 1,0 & 1,5 \\
\hline Myracrodruon urundeuva Allemão (Anacardiaceae) & KC 373 & 2 & 2 & 0,1 & 1,0 & 0,3 & 1,3 \\
\hline Anadenanthera colubrina (Vell.) Brenan (Mimosaceae) & KC 260 & 5 & 5 & 0,2 & 0,2 & 0,7 & 1,1 \\
\hline Mimosa tenuiflora (Willd.) Poir. (Mimosaceae) & KC 368 & 4 & 4 & 0,1 & 0,2 & 0,6 & 1,0 \\
\hline Amburana cearensis Allemão (Fabaceae) & AC 11 & 4 & 3 & 0,1 & 0,2 & 0,5 & 0,8 \\
\hline Guapira noxia (Netto) Lundell (Nyctaginaceae) & KC 353 & 2 & 2 & 0,1 & 0,3 & 0,3 & 0,6 \\
\hline Bauhinia cheilantha (Bong.) Steud. (Caesalpiniaceae) & KC 335 & 3 & 3 & 0,1 & 0,0 & 0,5 & 0,6 \\
\hline Harrisia adscendens (Gurke) Britton \& Rose (Cactaceae) & MR 837 & 5 & 2 & 0,2 & 0,1 & 0,3 & 0,6 \\
\hline Erytroxylum pungens O.E.Schulz (Erytroxylaceae) & MR 843 & 2 & 2 & 0,1 & 0,0 & 0,3 & 0,4 \\
\hline Senna macranthera (Collad.) Irwin \& Barneby (Caesalpiniaceae) & LP 96 & 2 & 2 & 0,1 & 0,0 & 0,3 & 0,4 \\
\hline Capparis flexuosa L. (Capparaceae) & LP 97 & 1 & 1 & 0,0 & 0,1 & 0,2 & 0,2 \\
\hline Pilosocereus pachycladus F.Ritter (Cactaceae) & MR 842 & 1 & 1 & 0,0 & 0,0 & 0,2 & 0,2 \\
\hline Cordia leucocephala Moric. (Boraginaceae) & KC 343 & 1 & 1 & 0,0 & 0,0 & 0,2 & 0,2 \\
\hline
\end{tabular}

recrutamento contínuo dessas populações uma vez que pode não estar ocorrendo recrutamento entre anos e sim de tempo de permanência longo em cada classe. Em Aspidosperma pyrifolium, com quase todos os indivíduos na primeira classe, é possível supor que essa população tenha sofrido algum tipo de corte no passado, considerando que em outros pontos próximos e com as mesmas características ambientais do hectare foram encontrados indivíduos com maiores diâmetros e alturas. Entretanto, a existência de indivíduos em todas as classes de diâmetro não seria suficiente para inferir resultados sobre o estabelecimento dessa população sem avaliar sua capacidade de produzir sementes viáveis.

Maiores considerações a respeito das populações amostradas com poucos indivíduos como Amburana cearensis, Anadenanthera colubrina, Commiphora leptophloeos, Myracrodruon urundeuva e Schinopsis brasiliensis são bastante limitadas. Todavia, vale ressaltar que diversos levantamentos na depressão sertaneja apontam que essas espécies apresentam naturalmente baixa densidade (Nascimento et al. 
2003). A literatura mostra que essas espécies tendem a apresentar maior densidade e porte em áreas com vegetação de caatinga mais úmidas (Pereira et al. 2002, Alcoforado Filho et al. 2003).

Os resultados das comparações entre os parâmetros fisionômicos em áreas da depressão sertaneja em situação de pediplano ou próxima a riacho/ serra mostram que em ambas é comum uma elevada densidade de plantas de pequeno porte e altura e que a separação fisionômica parece ser o resultado da presença de indivíduos de maiores diâmetros e alturas. É possível supor que pequenas mudanças nos habitats, como proximidade de serras e riachos, dentro de uma mesma unidade ambiental, possam explicar em parte a heterogeneidade fisionômica da caatinga sensu stricto.

\section{Literatura citada}

Alcoforado Filho, F.G., Sampaio, E.V.S.B. \& Rodal, M.J.N. 2003. Florística e fitossociologia de um remanescente de vegetação caducifólia espinhosa arbórea em Caruaru, Pernambuco. Acta Botanica Brasilica 17: 289-305.

Andrade-Lima, D. 1981. The caatingas dominium. Revista Brasileira de Botânica 4: 149-153.

Araújo, E.L., Sampaio, E.V.S.B. \& Rodal, M.J.N. 1995. Composição florística e fitossociologia de três áreas de Caatinga de Pernambuco. Revista Brasileira de Biologia 55: 595-607.

Ayres, M., Ayres Júnior, M., Ayres, D.L. \& Santos, A.S. 2000. BioEstat 2.0: Aplicações estatísticas nas áreas de ciências biológicas e médicas. Sociedade Civil Manirauá e CNPq, Belém.

Brasil. 1983. Projeto RADAMBRASIL. Folhas SC 24/25 - Aracaju/ Recife. Geologia, geomorfologia, pedologia, vegetação e uso potencial da terra. Levantamento de Recursos Naturais 30. Ministério das Minas e Energia. Secretaria Geral, Rio de Janeiro.

Cabrera, A.L. \& Willink, A. 1973. Biogeografia de America Latina. 2 ed. OEA, Washington.

Camacho, R.G.V. 2001. Estudo fitofisiográfico da Caatinga do Seridó - Estação Ecológica do Seridó, RN. Tese de Doutorado, Universidade de São Paulo, São Paulo.

Cronquist, A. 1981. An integrated system of classification of flowering plants. Columbia University Press, New York.

Embrapa. 1997. Manual de métodos de análise de solo. Empresa Brasileira de Pesquisa Agropecuária, Brasília.

Embrapa. 2003. Solos de Pernambuco. http://www.cnps. embrapa.br. (acesso em 10.07.2003).
Ferraz, E.M.N., Rodal, M.J.N. \& Sampaio, E.V.S.B. 2003. Physiognomy and structure of vegetation along an altitudinal gradient in the semi-arid region of Northeastern Brazil. Phytocoenologia 33: 71-92.

Instituto Nacional de Meteorologia. 2006. Balanço hídrico climatológico. http://www.inmet.gov.br. (acesso em 28.08.2006).

Lewis, G.P. 1998. Caesalpinia: revision of PoincianellaErythrostemon. Royal Botanic Gardens, Kew.

Loiola, M.I.B. 2001. Revisão taxonômica de Erythroxylum sect. Rhabdophyllum O. E. Schulz. Tese de Doutorado, Universidade Federal Rural de Pernambuco, Recife.

Lucena, M.F.A. 2001. Estudo taxonômico do gênero Croton (Crotonoideae, Euphorbiaceae), nas zonas do Litoral e da Mata do estado de Pernambuco, Brasil. Dissertação de Mestrado, Universidade Federal Rural de Pernambuco, Recife.

Medeiros, J.B.L.P. 1995. Florística e fitossociologia de uma área de caatinga localizada na fazenda Aracangá, Município de Capristano - CE. Monografia de Graduação, Universidade Federal do Ceará, Fortaleza.

Mueller-Dombois, D. \& Ellenberg, H. 1974. Aims and methods of vegetation ecology. John Willey \& Sons, New York.

Nascimento, A.R.T., Felfili, J.M. \& Meirelles, E.M. 2004. Florística e estrutura da comunidade arbórea de um remanescente de Floresta Estacional Decidual de encosta, Monte Alegre, GO, Brasil. Acta Botanica Brasilica 18: 659-669.

Nascimento, C.E.S., Rodal, M.J.N. \& Cavalcanti, A.C. 2003. Phytosociology of the remaining xerophytic woodland associated to an environmental gradient at the banks of the São Francisco River - Petrolina, Pernambuco, Brazil. Revista Brasileira de Botânica 26: 271-287.

Pereira, I.M., Andrade, L.A., Barbosa, M.R.V. \& Sampaio, E.V.S.B. 2002. Composição florística e análise fitossociológica do componente arbustivoarbóreo de um remanescente florestal no Agreste Paraibano. Acta Botanica Brasilica 16: 357-2002.

Rizzini, C.T. 1979. Tratado de fitogeografia do Brasil, v. 2: aspectos sociológicos e florísticos. Universidade de São Paulo, São Paulo.

Rodal, M.J.N. 1992. Fitossociologia da vegetação arbustivo-arbórea em quatro áreas de caatinga em Pernambuco. Tese de Doutorado, Universidade Estadual de Campinas, Campinas.

Rodal, M.J.N. \& Sampaio, E.V.S.B. 2002. A vegetação do bioma Caatinga. In: E.V.S.B. Sampaio; A.M. Giulietti, J. Virgínio \& C.F.L. Gamarra-Rojas (eds.). Vegetação e Flora da Caatinga. Associação Plantas do Nordeste / Centro Nordestino de Informações sobre Plantas, Recife, pp. 11-24. 
Rodal, M.J.N., Sampaio, E.V.S. \& Figueiredo, M.A. 1992. Manual sobre métodos de estudo florístico e fitossociológico - ecossistema Caatinga. Sociedade Botânica do Brasil, Brasília.

Sampaio, E.V.S.B. 1995. Overview of the Brazilian Caatinga. In: H.A. Mooney, S.H. Bullock \& E. Medina (eds.). Dry tropical forests. Cambridge University Press, Cambridge, pp. 35-63.

Sampaio, E.V.S.B. 1996. Fitossociologia. In: E.V.S.B. Sampaio, S.J. Mayo \& M.R.V. Barbosa (eds.). Pesquisa botânica nordestina: progresso e perspectivas. Sociedade Botânica do Brasil, Seção Regional de Pernambuco, Recife, pp. 203-230
Shepherd, G.J. 1994. FITOPAC. Manual do usuário. Departamento de Botânica, Universidade Estadual de Campinas, Campinas.

Souza, M.J.N., Martins, M.L.R., Soares, Z.M.L., Freitas Filho, M.R., Almeida, M.A.G., Pinheiro, F.S.A., Sampaio, M.A.B., Carvalho, G.M.B.S., Soares, A.M.L., Gomes, E.C.B. \& Silva, R.A. 1994. Redimensionamento da região semi-árida do Nordeste do Brasil. In: Conferência Nacional e Seminário LatinoAmericano de Desertificação. Fundação Esquel do Brasil, Fortaleza, pp. 1-24.

Veloso, H.P., Rangel Filho, A.L.R. \& Lima, J.C.A. 1991. Classificação da vegetação brasileira, adaptada a um sistema universal. IBGE, Rio de Janeiro. 